\title{
Feasibility study of nuclear transmutation by negative muon capture reaction using the PHITS code
}

\author{
Shin-ichiro Abe ${ }^{1 a}$, and Tatsuhiko Sato ${ }^{1}$ \\ ${ }^{1}$ Japan Atomic Energy Agency, Nuclear Science and Engineering Center, 2-4 Shirakata, Tokai-mura, Naka-gun, \\ Ibaraki 319-1195, Japan
}

\begin{abstract}
Feasibility of nuclear transmutation of fission products in high-level radioactive waste by negative muon capture reaction is investigated using the Particle and Heave Ion Transport code System (PHITS). It is found that about $80 \%$ of stopped negative muons contribute to transmute target nuclide into stable or short-lived nuclide in the case of ${ }^{135} \mathrm{Cs}$, which is one of the most important nuclide in the transmutation. The simulation result also indicates that the position of transmutation is controllable by changing the energy of incident negative muon. Based on our simulation, it takes approximately $8.5 \times 10^{8}$ years to transmute $500 \mathrm{~g}$ of ${ }^{135} \mathrm{Cs}$ by negative muon beam with the highest intensity currently available.
\end{abstract}

\section{Introduction}

Partitioning and transmutation (P\&T) have been investigated to reduce the radiotoxicity and storage time of the high-level radioactive waste (HLW) [1-5]. In the present reprocessing, $\mathrm{U}$ and $\mathrm{Pu}$ are recovered from spent fuel and HLW is vitrified. In the P\&T process, HLW is separated into below groups; minor metals (e.g., Ru, Rh, Pd), minor actinides (MA), Sr and Cs, and the other fission products (FPs). Minor metals are utilized as catalysts, material for electronic device and so on. MA are transmuted into stable of short-lived nuclei by neutron irradiation with nuclear fuels in the thermal reactor, fast reactor or accelerator driven sub-critical system (ADS). Sr and Cs are stabilized as a calcified waste form because they have high heat load, and the other FPs are vitrified.

After transmutation of MA, residual FPs become major cause for long-term repository of radioactive wastes. Therefore, transmutation of FP contributes further reduction of storage time. Several studies of the transmutation of FP have been performed $[4,5]$. Even though transmutation by thermal neutrons can be applied to some nuclides (e.g., $\left.{ }^{99} \mathrm{Tc},{ }^{129} \mathrm{I}\right)$, it is difficult to transmute FPs having small cross section for neutron capture reaction. Table 1 shows the thermal neutron capture cross section, content in spent fuel and half-life of typical FPs.

Negative muon has possibility to apply transmutation of FP through capture reaction. As a result of the weak interaction, a proton in the nucleus changes to a neutron and nucleus gains excitation energy:

$$
\mu^{-}+\mathrm{X}(N, Z) \rightarrow v_{\mu}+\mathrm{Y}(N+1, Z-1)^{*}+\left(m_{\mu}+m_{\mathrm{X}}-m_{\mathrm{Y}}\right) \mathrm{MeV}
$$

\footnotetext{
${ }^{\mathrm{a}}$ Corresponding author: abe.shinichiro@jaea.go.jp
} 
Table 1. Thermal neutron capture cross section [6], content in spent fuel and half-life [7] of typical FPs.

\begin{tabular}{|c|r|r|r|}
\hline $\begin{array}{c}\text { Radio } \\
\text { isotope }\end{array}$ & $\begin{array}{c}\text { Cross section of } \\
\left(\mathbf{n}_{\text {th }}, \boldsymbol{\gamma}\right) \text { [barn] }\end{array}$ & $\begin{array}{c}\text { Content in spent } \\
\text { fuel [kg/ton] }\end{array}$ & $\begin{array}{c}\text { Half-life } \\
\text { [year] }\end{array}$ \\
\hline${ }^{79} \mathrm{Se}$ & 50 & 0.006 & $2.95 \mathrm{e}+5$ \\
\hline${ }^{90} \mathrm{Sr}$ & 0.0101 & 0.6 & 28.79 \\
\hline${ }^{93} \mathrm{Zr}$ & 2.239 & 1 & $1.61 \mathrm{e}+6$ \\
\hline${ }^{99} \mathrm{Tc}$ & 23.59 & 1 & $2.11 \mathrm{e}+5$ \\
\hline${ }^{107} \mathrm{Pd}$ & 9.241 & 0.3 & $6.5 \mathrm{e}+6$ \\
\hline${ }^{126} \mathrm{Sn}$ & 0.09 & 0.03 & $2.30 \mathrm{e}+5$ \\
\hline${ }^{129} \mathrm{I}$ & 30.31 & 93.3 & $1.57 \mathrm{e}+7$ \\
\hline${ }^{135} \mathrm{Cs}$ & 8.3 & 223 & $2.3 \mathrm{e}+6$ \\
\hline${ }^{137} \mathrm{Cs}$ & 0.27 & 659 & 30.08 \\
\hline
\end{tabular}

where $m_{n}, m_{\mathrm{X}}$ and $m_{\mathrm{Y}}$ are the rest mass of negative muon and nucleus before and after negative muon capture reaction, respectively. The unique feature of negative muon capture reaction is the controllability of capture position. A negative muon is captured by nucleus just before it stops.

Recently, negative muon capture process has been implemented into the Particle and Heavy Ion Transport code System (PHITS) [8]. In the present work, the production ratio of secondary nucleus from negative muon capture reaction on typical FPs are calculated using PHITS. The controllability of the position of transmutation is demonstrate. After that, the feasibility of nuclear transmutation by negative muon capture reaction for FPs is discussed.

\section{Model of negative muon capture reaction in PHITS}

At first, a negative muon is captured by an atom and the muonic atom is formed. The captured negative muon cascades down to the $1 \mathrm{~s}$ orbit while emitting characteristic X-rays. After that, the negative muon is decayed in the 1 s orbit or captured by the nucleus. As shown in eq. (1), a proton in the nucleus captures the negative muon and it becomes a neutron.

In PHITS, the muonic atom cascade program [9] is employed to simulate the cascade of the captured negative muon in the atomic orbits. The decay rate of negative muon is derived by using the Huff factor taken from [10] or calculated by fitting function of [11]. The nuclear capture rate of negative muon is calculated by the Goulard-Primakoff formula [12]. For light atoms $(Z<10)$, the measured nuclear capture rate taken from [10] are tableted and used.

When a negative muon is captured by a nucleus, the excitation energy of the nucleus is sampled from excitation function proposed in [13]. A proton in the nucleus is selected randomly and is replaced to a neutron added the excitation energy. After that, the time evolution of nucleons is calculated by JAERI Quantum Molecular Dynamics (JQMD) model [14] and evaporation process is calculated by Generalized Evaporation Model (GEM) [15], respectively.

Figure 1 shows the differential cross section of secondary neutron from negative muon capture on ${ }^{40} \mathrm{Ca}$ and ${ }^{208} \mathrm{~Pb}$. The experimental data $[16,17]$ are also plotted. PHITS is in good agreement with experimental data at low emission energies for ${ }^{40} \mathrm{Ca}$ target, although it tends to overestimate for ${ }^{208} \mathrm{~Pb}$. At emission energies above $30 \mathrm{MeV}$, PHITS underestimate neutron production for both targets. It was reported that high energy neutron is generated from $\mu \rightarrow q \bar{q} v_{\mu}$ decay mechanism [11], which does not considered in PHITS. Figure 2 compares the neutron multiplicity from negative muon capture on ${ }^{\text {nat }} \mathrm{Ag}$ and ${ }^{208} \mathrm{~Pb}$ with the experimental data [18]. PHITS describes the neutron multiplicity well over the whole range for ${ }^{\text {nat }} \mathrm{Ag}$, whereas PHITS overestimate the number of emitted neutron from ${ }^{208} \mathrm{~Pb}$. It is expected that PHITS describes the production of secondary nuclei from negative muon 

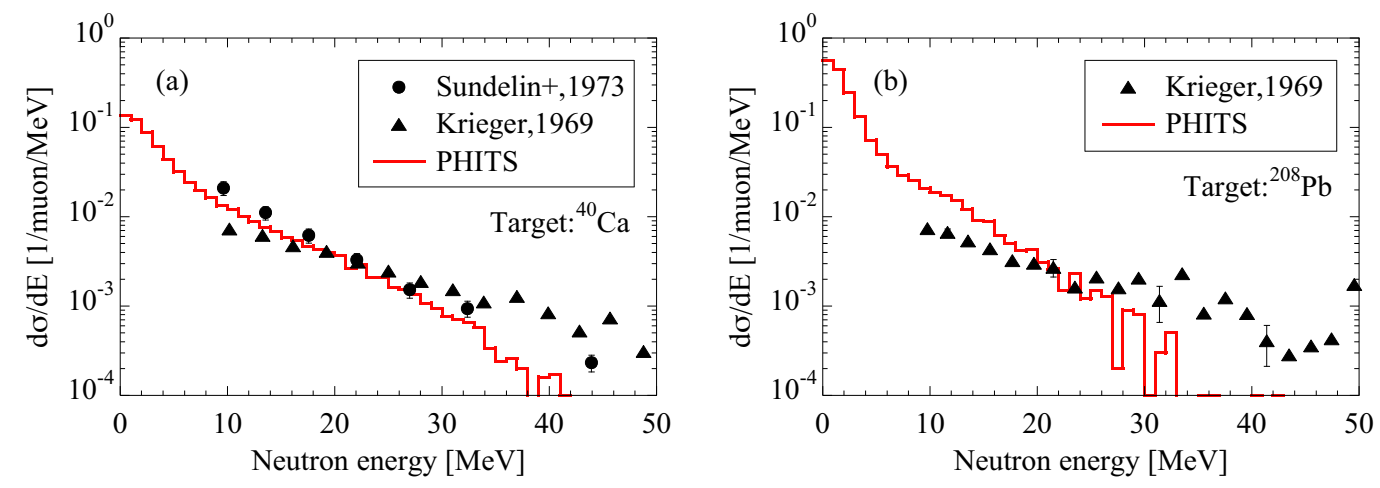

Figure 1. Differential cross section of secondary neutron from negative muon capture on (a) ${ }^{40} \mathrm{Ca}$ and (b) ${ }^{208} \mathrm{~Pb}$. The experimental data are taken from $[16,17]$.
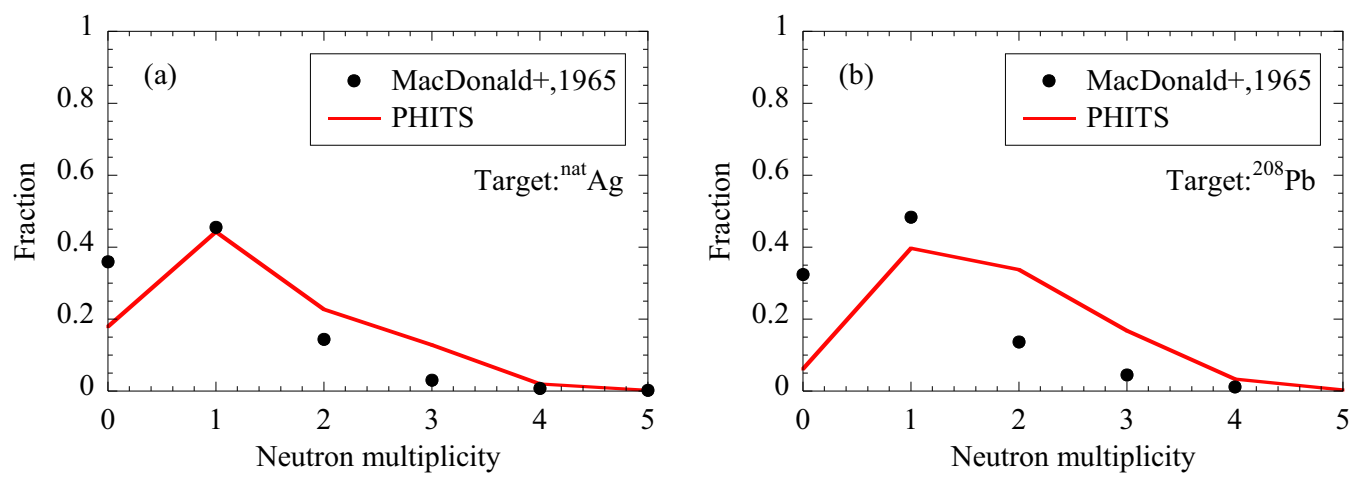

Figure 2. Neutron multiplicity from negative muon capture on (a) ${ }^{\text {nat }} \mathrm{Ag}$ and (b) ${ }^{208} \mathrm{~Pb}$. The experimental data is taken from [18].

capture on FPs within reasonable range because the atomic number of target FPs is closer to Ag rather than $\mathrm{Pb}$.

\section{Simulation of nuclear transmutation for FPs}

Nuclear transmutation by negative muon capture is simulated for ${ }^{79} \mathrm{Se},{ }^{90} \mathrm{Sr},{ }^{93} \mathrm{Zr},{ }^{99} \mathrm{Tc},{ }^{107} \mathrm{Pd},{ }^{126} \mathrm{Sn}$, ${ }^{129} \mathrm{I},{ }^{135} \mathrm{Cs}$ and ${ }^{137} \mathrm{Cs}$. Table 2 shows the capture rate, the decay rate, and mean productions of neutron and proton for each target nuclide. About $5 \%$ of stopped negative muons decay in orbit for all target nuclide and hence they do not contribute to transmutation. The number of event for proton emission is less than $8 \%$, thus the target nucleus with an atomic number of $Z$ is mostly transmuted to nucleus with an atomic number of $Z-1$. Table 3 and 4 show the production ratio and decay chain of secondary nuclides produced by negative muon capture reactions on ${ }^{135} \mathrm{Cs}$ and ${ }^{137} \mathrm{Cs}$ as examples. It is found that $12.5 \%$ of ${ }^{135} \mathrm{Cs}$ become ${ }^{135} \mathrm{Xe}$ and they return to ${ }^{135} \mathrm{Cs}$ by $\beta$ - decay. As a result, $83.4 \%$ of ${ }^{135} \mathrm{Cs}$ become to stable or short-lived nuclides. In the case of ${ }^{137} \mathrm{Cs}, 4.6 \%$ of ${ }^{137} \mathrm{Cs}$ return to the original nuclide and $26.7 \%$ of ${ }^{137} \mathrm{Cs}$ are transmuted to ${ }^{135} \mathrm{Xe}$ and they become to ${ }^{135} \mathrm{Cs}$ having longer half-life than original nuclide. Consequently, $61.5 \%$ of ${ }^{137} \mathrm{Cs}$ become to stable or short-lived nuclides. For the other FPs, approximately $66 \%$ to $87 \%$ of FPs are transmuted to stable or short-lived nuclides by negative muon capture reaction.

In order to demonstrate the controllability of the position of transmutation, irradiation of negative muon beam on ${ }^{135} \mathrm{Cs}$ target are simulated by PHITS. The momentum of incident negative muon is 60 
Table 2. Capture rate, decay rate, and mean productions of neutron and proton for each target nuclide.

\begin{tabular}{|c|c|c|c|c|}
\hline \multirow{2}{*}{$\begin{array}{l}\text { Target } \\
\text { nuclide }\end{array}$} & \multirow{2}{*}{$\begin{array}{c}\text { Capture rate } \\
{[1 / \text { muon }]}\end{array}$} & \multirow{2}{*}{$\begin{array}{c}\text { Decay rate } \\
{[1 / \text { muon }]}\end{array}$} & \multicolumn{2}{|c|}{ Mean production [1/muon] } \\
\hline & & & neutron & proton \\
\hline${ }^{79} \mathrm{Se}$ & 0.925 & 0.075 & 1.197 & 0.059 \\
\hline${ }^{90} \mathrm{Sr}$ & 0.935 & 0.065 & 1.540 & 0.048 \\
\hline${ }^{93} \mathrm{Zr}$ & 0.948 & 0.052 & 1.413 & 0.068 \\
\hline${ }^{99} \mathrm{Tc}$ & 0.949 & 0.051 & 1.432 & 0.070 \\
\hline${ }^{107} \mathrm{Pd}$ & 0.962 & 0.038 & 1.325 & 0.081 \\
\hline${ }^{126} \mathrm{Sn}$ & 0.940 & 0.060 & 1.622 & 0.008 \\
\hline${ }^{129} \mathrm{I}$ & 0.959 & 0.041 & 1.436 & 0.062 \\
\hline${ }^{135} \mathrm{Cs}$ & 0.959 & 0.041 & 1.401 & 0.063 \\
\hline${ }^{137} \mathrm{Cs}$ & 0.954 & 0.046 & 1.632 & 0.052 \\
\hline
\end{tabular}

Table 3. Production ratio and decay chain of secondary nuclides from negative muon capture reactions on ${ }^{135} \mathrm{Cs}$

\begin{tabular}{|c|c|c|c|c|}
\hline $\begin{array}{l}\text { Product } \\
{[1 / \text { muon }]}\end{array}$ & 0.129 & 0.224 & 0.393 & 0.125 \\
\hline $\begin{array}{c}\text { Decay } \\
\text { chain } \\
\text { (Half-life) }\end{array}$ & $\begin{array}{c}{ }^{132} \mathrm{Xe} \\
\text { (stable) }\end{array}$ & $\begin{array}{c}{ }^{133} \mathrm{Xe} \\
(5.25 \mathrm{~d}) \\
\nabla \\
{ }^{133} \mathrm{Cs} \\
\text { (stable) }\end{array}$ & $\begin{array}{c}{ }^{134} \mathrm{Xe} \\
\text { (stable) }\end{array}$ & $\begin{array}{c}{ }^{135} \mathrm{Xe} \\
(9.14 \mathrm{~h}) \\
\boldsymbol{\nabla} \\
{ }^{135} \mathrm{Cs} \\
(2.3 \mathrm{e}+6 \mathrm{y}) \\
\boldsymbol{\nabla} \\
{ }^{135} \mathrm{Ba} \\
\text { (stable) }\end{array}$ \\
\hline
\end{tabular}

Table 4. Production ratio and decay chain of secondary nuclides from negative muon capture reactions on ${ }^{137} \mathrm{Cs}$

\begin{tabular}{|c|c|c|c|c|}
\hline $\begin{array}{c}\text { Product } \\
\text { [1/muon] }\end{array}$ & 0.163 & 0.267 & 0.388 & 0.046 \\
\hline & ${ }^{134} \mathrm{Xe}$ & ${ }^{135} \mathrm{Xe}$ & ${ }^{136} \mathrm{Xe}$ & ${ }^{137} \mathrm{Xe}$ \\
$($ stable $)$ & $(9.14 \mathrm{~h})$ & $($ stable $)$ & $(3.18 \mathrm{~m})$ \\
$\begin{array}{c}\text { Decay } \\
\text { chain }\end{array}$ & & $\boldsymbol{\nabla}$ & & $\boldsymbol{\nabla}$ \\
(Half-life) & & ${ }^{135} \mathrm{Cs}$ & & ${ }^{137} \mathrm{Cs}$ \\
& & $(2.3 \mathrm{e}+6 \mathrm{y})$ & & $(30.1 \mathrm{y})$ \\
& & $\boldsymbol{\nabla}$ & & $\boldsymbol{\nabla}$ \\
& & ${ }^{135} \mathrm{Ba}$ & & ${ }^{137} \mathrm{Ba}$ \\
$($ stable $)$ & & $($ stable $)$ \\
\hline
\end{tabular}

$\mathrm{MeV} / \mathrm{c}$ with the deviation of $5 \%$ (i.e., it corresponds to the incident energy of $16 \pm 1.5 \mathrm{MeV}$ ) which desired momentum for muon beam of MuSIC [19] at RCNP. The shape of target is cylinder with a radius of $1 \mathrm{~cm}$ and a length of $4 \mathrm{~cm}$. A lead moderator with a thickness of $1.5 \mathrm{~mm}$ is put in front of the target. The energy loss of the incident negative muon in the moderator is about $6 \mathrm{MeV}$. The energy straggling and the angular straggling are considered in the simulation. Figure 3 shows the depth distribution of produced nucleus with or without the moderator. The central depth of produced nucleus is $0.7 \mathrm{~cm}$ and $1.5 \mathrm{~cm}$, which correspond to the range of the negative muon in ${ }^{135} \mathrm{Cs}$ target having the energy of $10 \mathrm{MeV}$ and $16 \mathrm{MeV}$, respectively. The spatial deviation of produced nucleus is $0.35 \mathrm{~cm}$ 


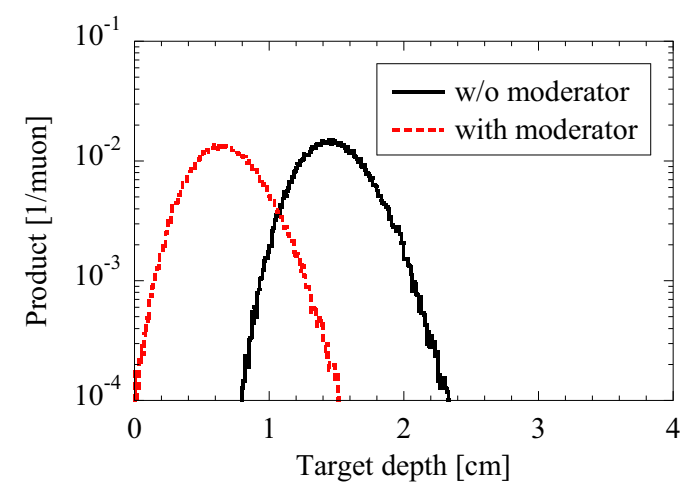

Figure 3. Depth distribution of produced Xe with or without the lead moderator.

due to the deviation of the incident momentum. This result indicates that the energy distribution of incident negative muon is directly reflected on the spatial distribution of secondary nuclei. The position of transmutation is controllable by using moderator or changing beam energy.

Finally, the condition of negative muon source required for transmutation is discussed. The time required to transmute of all FPs can be estimated briefly as follows:

$$
t=N_{\mathrm{A}} \times \frac{g}{M} \times \frac{\alpha}{\phi}
$$

where $N_{\mathrm{A}}$ is the Avogadro's number, $g$ is the content of the FP in spent fuel, $M$ is the mass number of the target FP, $\alpha$ is the transmutation ratio to stable or short-lived nucleus, and $\phi$ is the intensity of negative muon. Even though the intensity of negative muon reaches $10^{8}$ muons/sec which corresponds to the muon intensity of MuSIC at RCNP, it takes about $8.5 \times 10^{8}$ year to transmute $500 \mathrm{~g}$ of ${ }^{135} \mathrm{Cs}$. Therefore, much higher intensity of negative muon source is required for transmutation by negative muon capture reaction.

\section{Conclusions}

Nuclear transmutation of FPs by negative muon capture reaction was simulated by PHITS. It was found that target nuclide with an atomic number of $Z$ is mostly transmuted to nuclide with an atomic number of $Z-1$. As a result, for our investigated case, $61 \%$ to $87 \%$ of stopped negative muons contribute to transmute target nuclide into stable or short-lived nuclide. In the case of ${ }^{137} \mathrm{Cs}, 26.7 \%$ of ${ }^{137} \mathrm{Cs}$ is transmuted into ${ }^{135} \mathrm{Cs}$ which have longer life-time than original nuclide. The irradiation of negative muon beam was also simulated. The result indicates that the position of transmutation by negative muon capture is controllable by changing incident energy of negative muon. From a rough estimate, it takes about $8.5 \times 10^{6}$ year to transmute $500 \mathrm{~g}$ of ${ }^{135} \mathrm{Cs}$ with the negative muon intensity of $10^{8}$ muons/sec. We conclude that much higher intensity of negative muon source is required for transmutation by negative muon capture reaction.

\section{References}

1. OECD/NEA, NEA Report No.6894 (2011).

2. K. Nishihara et al., J. Nucl. Sci. Technol., 45, 84 (2012).

3. K. Tsujimoto et al., J. Nucl. Sci. Technol., 41, 21 (2004).

4. T. Wakabayashi et al., Prog. Nucl. Energy, 32, 555 (1998).

5. W. S. Yang et al., Nucl. Sci. Eng., 146, 291 (2004). 
6. K. Shibata et al., J. Nucl. Sci. Technol., 48, 1 (2011).

7. Japanese Nuclear Data Committee, Chart of the Nuclides (2014).

8. T. Sato et al., J. Nucl. Sci. Technol., 50, 913 (2013).

9. V. R. Akylas et al., Comp. Phys. Comm., 15, 291 (1978).

10. T. Suzuki et al., Phys. Rev. C, 35, 2212 (1987).

11. M. V. Kossov, Eur. Phys. J. A, 33, 7 (2007).

12. B. Goulard et al., Phys. Rev. C, 10, 2034 (1974).

13. P. Singer et al., Il Nuovo Cimento, 23, 669 (1962).

14. K. Niita et al., Phys. Rev. C, 52, 2620 (1995).

15. S. Furihata, Nucl. Instr. and Meth. in Phys. Res. B, 171, 251 (2000).

16. R. M. Sundelin et al., Phys. Rev. C, 7, 1037 (1973).

17. M. H. Krieger, Ph.D Thesis, Columbia Univ. (1969).

18. B. MacDonald et al., Phys. Rev., 139, B1253 (1965).

19. Y. Hino et al., Nucl. Phys. B Proc. Suppl., 253-255, 206-207 (2014). 